\title{
Pyrethroid Resistance in Insects: Genes, Mechanisms, and Regulation
}

\author{
Nannan Liu \\ Department of Entomology and Plant Pathology \\ Insect Molecular Toxicology and Physiology Program \\ Auburn University, Auburn, \\ Alabama, \\ USA
}

\section{Introduction}

Insecticides are the most important component in insect pest-control efforts worldwide. Pyrethroids are among the insecticides that have been most widely used for this purpose for many years because of their safe, cheap, effective and long-lasting nature (Bulter et al. 2011). However, the widespread development of insecticide resistance, especially pyrethroid resistance, and the fact that resistance to an insecticide generally confers cross-resistance to other insecticides has become an immense practical problem challenging the control of agriculturally, economically, and medically important insect pests and resulting in the rise of insect vector-borne diseases in many parts of the world (Zaim 2002; Bulter 2011). Insecticide resistance is a pre-adaptive phenomenon, in the sense that prior to an organism's (e.g., a mosquito's) exposure to insecticides, rare individuals (resistant individuals) carrying one or more possible resistance genes (or an altered [varied] genome) already exist, allowing the organism to survive exposure to the insecticides (WHO 1957). A large number of studies have indicated that multiple resistance mechanisms or genes are involved in the development of insecticide resistance in many insect species, including mosquitoes and house flies (Raymond et al. 1989; Hemingway et al. 2002; 2004; Liu and Scott 1995; 1996; 1997; 1998; Liu and Yue 2000; 2001; Ranson et al. 2002; Liu et al. 2005; Vontas et al. 2005; Xu et al. 2005; Liu et al. 2007; Zhu and Liu 2008; Zhu et al. 2008a; b; Liu et al. 2011). Characterization of the molecular mechanisms and genes involved in insecticide resistance has therefore been fundamental in understanding the development of resistance and in practical applications such as designing novel strategies to prevent or minimize the spread and evolution of resistance development and control insect pests.

Three major mechanisms are involved in insecticide resistance: (1) increased metabolic detoxification of insecticides; (2) decreased sensitivity of the target proteins on which an insecticide acts, known as target site insensitivity; and (3) decreased cuticular penetration/or increased sequestration/storage. Gene overexpression, amplification, and structural mutations have been linked to insecticide resistance mechanisms in some insects, while transcriptional overexpression of genes in resistant insects appears to be a common determining event in the evolution of resistance in insects generally. Regulatory interaction 
among different mechanisms and/or genes in resistance offers a tantalizing hint regarding precisely how these high levels of resistance in insects may be developed. There is, however, an urgent need to improve our understanding of the mechanisms governing resistance development, which is essential if we are to develop new and more effective strategies to circumvent and/or delay resistance development, control resistant insect pests, and reduce the prevalence of insect vector-borne diseases. In this review, I will focus on the genes and mechanisms, along with their interactions and regulation, that lead to the development of insecticide resistance and discuss the potential impact of a better understanding of resistance mechanisms on the basic and practical aspects of research into how mosquitoes and house flies develop a resistance to insecticides.

\section{Metabolic detoxification-mediated resistance}

The mechanism of increased detoxification contributes to a decrease in the effective dose of insecticides available at the target site (Scott 1990; 1999; Feyereisen 1995; Pasteur 1996). The products of three gene families, cytochrome P450 monooxygenases (cytochrome P450s), hydrolases, and glutathione S-transferases (GSTs), are primarily implicated in the detoxification of insecticides. In the mosquito, for example, the detoxification of insecticides in the mosquito involves cytochrome P450s (P450s), esterases, and GSTs (Feyereisen 2005; Ranson and Hemingway 2005; Oakeshott et al. 2005). Insect cytochrome P450s in particular are known to play an important role in detoxifying insecticides (Scott 1999; Feyereisen 2005). Transcriptional up-regulation of $\mathrm{P} 450$ s results in an increase in P450 proteins and P450 activities, which, in turn, trigger the development of insecticide resistance. Esterases are a group of heterogeneous enzymes present in most organisms. Although an increase in the DNA amplification of esterases has been associated with esterase-mediated insecticide resistance (Hemingway and Karunaratne 1998; Small and Hemingway 2000), non-elevated esterase levels have also been identified in mosquitoes exhibiting insecticide resistance (Whyard et al. 1995). GSTs are soluble dimeric proteins involved in the metabolism, detoxification, and excretion of a large number of endogenous and exogenous compounds (Ranson and Hemingway 2005). The up-regulation of GST genes has been identified in the mosquito Anopheles gambiae, which is resistant to pyrethroids (Ortelli et al. 2003). In other insect species such as the diamondback moth and the rice brown plant hopper, upregulation of GST genes is also known to be involved in insecticide resistance (Vontas et al. 2005). Even though the detoxification gene families involved in insecticide resistance are known, however, little is known about how many genes in each family are directly involved in resistance in a resistant insect. The molecular mechanisms involved in insecticide resistance in general, and the regulation of up-regulated P450 genes, GSTs, and esterases in particular, are not yet clear.

\section{P450-mediated pyrethroid resistance}

Cytochrome P450s constitute one of the largest gene superfamilies in all living organisms, including mammals, fish, arthropods, fungi, plants, and bacteria, and they are known to perform a large number of highly diverse physiological and biochemical functions. In insects, more than 1700 P450s have so far been identified (Nelson 2009, http://drnelson.uthsc.edu/CytochromeP450.html). Cytochrome P450s have long been of particular interest because they are critical for the detoxification and/or activation of 
xenobiotics such as drugs, pesticides, plant toxins, chemical carcinogens and mutagens. They are also involved in metabolizing endogenous compounds such as hormones, fatty acids, and steroids. Insect cytochrome P450s are known to play an important role in the detoxification and/or activation of xenobiotics such as insecticides (Scott 1999; Feyereisen, 2005) and plant toxins (Berenbaum 1991; Schuler 1996), leading to the development of resistance to insecticides (Carino et al. 1994; Liu and Scott 1997; 1998; Feyereisen 2005; Zhu et al. 2008a; Komagata et al. 2010; Liu et al. 2011) and tolerance to plant toxins (Li et al. 2002; Wen et al. 2003). Basal and up-regulation of P450 gene expression can significantly affect the disposition of xenobiotics or endogenous compounds in the tissues of organisms and thus alter their pharmacological/toxicological effects (Pavek and Dvorak 2008). Increased P450-mediated detoxification has been found in many insect species associated with enhanced metabolic detoxification of insecticides, as evidenced by the increased levels of P450 proteins and P450 activity that result from constitutively transcriptional overexpression of P450 genes in insecticide resistant insects. In addition, some insect $\mathrm{P} 450$ genes can be induced by exogenous compounds. Recently, our group has identified that multiple P450 genes are overexpressed in insecticide resistant house flies Musca domestica and mosquitoes Culex quinquefasciatus through both constitutive overexpression and induction by pyrethroids ((Zhu and Liu 2008; Zhu et al. 2008a; b; Liu et al. 2011), suggesting that constitutive overexpression and induction are key factors for increased levels of detoxification of insecticides and insecticide resistance. Nevertheless, although their importance in insect physiology and toxicology is widely recognized, there are enormous gaps in our knowledge of insect P450s. In particular, their precise role in the regulation processes that govern the evolution of insecticide resistance, which typically requires the interaction of multiple genes, has not yet been determined. As more P450 sequences become available, our understanding of the roles of P450s in physiological and toxicological processes should be improved.

The initial characterization of the importance of metabolic detoxification in insecticide resistance has primarily been documented in many insect species on the basis of synergistic studies. These studies have revealed that resistance to insecticides is decreased by piperonyl butoxide (PBO), the inhibitor of cytochrome P450 monooxygenases. In a series of studies in our laboratory designed to investigate how pyrethroid resistance develops, a house fly strain ALHFG0 and a mosquito strain $\mathrm{HAmCqG0}$ were collected from sites in Alabama and further selected with permethrin for 6 and 8 generations, respectively, generating the strains of ALHF and HAmCqG8 (Liu and Yue 2000; 2001; Liu et al. 2004a; Xu et al. 2006a; Li et al. 2010). Both permethrin selected house fly and mosquito strains achieved very high levels of resistance compared with their parental strains. Synergism studies found that permethrin resistance in ALHF house flies and HAmCq mosquitoes (both- the parental strains and their selected offspring) was largely suppressed by piperonyl butoxide (PBO), indicating that P450 monooxygenase-mediated detoxification may be one of the major mechanisms involved in the development of pyrethroid resistance in these insect species (Liu and Yue 2000; Xu et al. 2005).

\section{Constitutive overexpression of P450 genes in pyrethroid resistant house flies and mosquitoes}

The increase in the levels of P450 proteins and P450 activities that results from the constitutive overexpression of $\mathrm{P} 450$ genes in insecticide resistant insects has been clearly 
implicated in the development of resistance to insecticides. Based on this critical finding, our group has examined the expression profiles of $\mathrm{P} 450$ genes from both house flies and mosquito $C x$. quinquefasciatus, comparing susceptible and resistant populations and the field parental population and their permethrin selected offspring. The differential expression patterns of all eight P450 genes, CYP4G13v1, CYP4D4v2, CYP4G2, CYP6A5v2, CYP6A36, CYP6A37, CYP6A38, and CYP28B1, were characterized for resistant ALHF and susceptible CS house fly strains and for different types of tissues using Northern blot analyses and quantitative real-time PCR (qRT-PCR) (Zhu and Liu 2008; Zhu et al. 2008a; b). No significant difference in the expression of CYP4G13v1, CYP4D4v2, CYP4G2, CYP6A37, CYP6A38, and CYP28B1 between resistant ALHF and susceptible CS and aabys flies was observed, but CYP6A5v2 and CYP6A36 showed significant constitutive overexpression in the resistant ALHF strain. Four cytochrome P450 cDNAs, CYP6AA7, CYP9J40, CYP9J34, and CYP9M10, were also isolated from mosquitoes, Cx. quinquefasciatus (Liu et al. 2011) and their expression was compared for three different mosquito populations bearing different resistance phenotypes, ranging from susceptible (S-Lab), through intermediate (HAmCqG0, the field parental population) to highly resistant (HAmCqG8, the $8^{\text {th }}$ generation of permethrin selected offspring of $\left.\mathrm{HAmCq}^{\mathrm{G} 0}\right)$. A strong correlation was found for all $\mathrm{P} 450$ gene expression with the levels of resistance and following permethrin selection at the larval stage of mosquitoes, with the highest expression levels identified in $\mathrm{HAmCqG8}$, suggesting the importance of CYP6AA7, CYP9J40, CYP9J34, and CYP9M10 in the permethrin resistance of larva mosquitoes.

Another important feature of insect P450 genes is that they may vary with regard to the tissues where they are expressed in response to physiological and environmental stimulators. In insects, the midgut and fat body tissues are generally considered to be the primary detoxification organs where most insect detoxification P450s are expressed (Hodgson 1985; Scott et al. 1998). The tissue specific expression of two P450 genes, CYP6A36 and CYP6A5v2, from house flies and CYP6AA7 from Cx. quinquefasciatus was examined because these genes overexpressed not only in resistant insects but also in adult stages. Although the expression of CYP6A5v2 was observed to be significantly higher in the abdominal tissue in both the susceptible CS and aabys flies and the resistant ALHF flies compared with their head+thorax tissues, this overexpression was far greater in both sets of tissues for resistant ALHF flies than in the tissues of susceptible flies (Zhu and Liu 2008). The expression of CYP6A36 was not significantly different between the head+thorax and abdomen tissues of the CS strain; lower in the head+thorax tissue than in the abdomen tissue of ALHF; and significantly higher in both tissues of the ALHF strain than in the CS strain (Zhu et al. 2008a). Significant overexpression of CYP6AA7 in resistant HAmCqG8 mosquitoes was found in all three types of tissues, head, thorax, and abdomen (Liu et al. 2011) compared with susceptible S-Lab mosquitoes, with the highest levels expressed being in the abdomen tissue. As midgut and most fat body components are located in the abdomen of insects and are known to be of primary importance in detoxification-related functions, the overexpression of the $3 \mathrm{P} 450$ genes, CYP6A5v2 and CYP6A36 in house flies and CYP6AA7 in mosquitoes, specifically in the abdomen tissues of resistant insect populations, suggests the importance of these genes in increasing the metabolic detoxification of insecticides in house flies and mosquitoes. However, because midgut and fat body tissues are not exclusively found in the abdomen, further dissection of detoxification-related tissues (such as midgut and fat 
body) is needed to pinpoint the precise location for the overexpression of these resistance related genes.

\section{Induction of P450 genes in response to permethrin exposure in house flies and mosquitoes}

Another characteristic of some insect P450 genes is that their expression can be induced by exogenous and endogenous compounds (Feyereisen 2005), a phenomenon known as induction. It has been suggested that many chemical inducers may act as substrates for the $\mathrm{P} 450$ s that they induce and that the induction of the P450s by the substrates will, in turn, reduce the effects of the substrates by enhancing substrate metabolism (Okey 1990). The involvement of the induction of P450s and their activities in the adaptation of insects to their environment and the development of insecticide resistance was proposed by Terriere (1983; 1984), who argued that while all insects probably possessed some capacity to detoxify insecticides and xenobiotics, the degree to which they can metabolize and detoxify these highly toxic chemicals was of considerable importance to their survival in a chemically unfriendly environment (Terriere 1984). Both constitutively increased expression (overexpression) and induction of P450s are thought to be responsible for increased levels of detoxification of insecticides. Multiple P450 genes induced in insects in response to host plant allelochemicals or secondary products have been extensively studied and are fairly well documented in terms of their function in the adaptation of insects in "animal-plant warfare" (Gonzalez and Nebert 1990) and in the co-evolution of insects and plants (Li et al. 2002). In contrast, P450 gene induction in response to insecticide resistance is less well understood.

The induction profiles of P450 genes in house flies and mosquitoes have been characterized in our laboratory. Dose range, time course, and P450 gene induction assays on $C x$. quinquefasciatus showed a clear time- and dose -dependent response of mosquito P450s to permethrin (Liu et al. 2011). No significant induction was detected in the expression of CYP6AA7, CYP9J34, and CYP9M10 in susceptible S-Lab mosquitoes, but various levels of induction were identified in the field parental mosquitoes $\mathrm{HAmCq \textrm {G } 0}$ and their permethrin selected offspring HAmCqG8. No significant expression of CYP6AA7 was detected in $\mathrm{HAmCqG0}$ that had been treated with either acetone alone (control) or with permethrin at 24 h. However, in the HAmCqG8 strain an initial induction of CYP6AA7 ( 1.5-fold) was found in mosquitoes that had been treated with a $\mathrm{LC}_{10}$ concentration of permethrin and a marked induction ( 4.5-fold) in those treated with the permethrin at a concentration of $\mathrm{LC}_{50}$. Levels of CYP9J34 RNA in HAmCqG0 were readily induced by a $\mathrm{LC}_{10}$ permethrin concentration, reaching a maximum ( 1.7-fold) for a $L_{50}$ permethrin concentration, with no further significant induction up to $\mathrm{LC}_{90}$. The induction of CYP9J34 was even more evident in the HAmCqG8 strain than in their parental $\mathrm{HAmCqG0}$, with an induction peak of $\sim 2.7$-fold at a permethrin concentration of $\mathrm{LC}_{50}$. A similar induction pattern was found for CYP9M10 in $\mathrm{HAmCqG0}$ and $\mathrm{HAmCqG8}$.

Comparable results for the time- and dose -dependent induction by permethrin on P450 gene expression have also been identified in house flies (Zhu et al. 2008b). The expression patterns of eight P450 genes, CYP4G13v1, CYP4D4v2, CYP4G2, CYP6A5v2, CYP6A36, CYP6A37, CYP6A38, and CYP28B1 in response to permethrin treatment in resistant ALHF and susceptible CS and aabys house flies were characterization by treating 2-day old adult house flies with permethrin. Three P450 genes, CYP4D4v2, CYP4G2, and CYP6A38, were co- 
up-regulated by permethrin treatment in permethrin resistant ALHF house flies with a $\mathrm{LD}_{50}$ dose and a $24 \mathrm{~h}$ time interval (Zhu et al. 2008b). No significant induction in the expression of these three P450 genes was found in susceptible house flies that had either been treated with acetone alone or with permethrin solution in acetone compared with untreated house flies. Similarly, no significant induction was obtained in acetone treated ALHF house flies compared with their untreated counterparts. However, all three of these genes were induced at various levels in permethrin treated ALHF house flies compared with untreated or acetone treated flies. There was a marked induction of CYP4D4v2 and CYP6A38 mRNA in permethrin treated ALHF house flies, whereas a low level of induction for CYP4G2 was detected in the permethrin treated ALHF house flies (Zhu et al. 2008b).

The significant induction of the P450 genes only in the field resistant and/or permethrin selected highly resistant strains of both house flies and mosquitoes strongly suggests the importance of P450 genes in the resistant insects, particularly with regard to their response to permethrin treatment. Taken together, these results indicate that multiple P450 genes are up-regulated in insecticide resistant insects through both constitutive overexpression and induction mechanisms, thus increasing the overall expression levels of P450 genes.

\section{Sodium channel mutation-mediated target-site insensitivity in pyrethroid resistance}

The mechanism of decreased target site sensitivity contributes to the ineffective binding of a given dose of inscticides (Scott 1990; 1999; Feyereisen 1995; Pasteur and Raymond 1996). Target-site insensitivity results from the structural modification or mutation (point mutation) of the target proteins that the insecticides act upon. Point mutations in the target protein cause a reduction in the nervous system's response to insecticides (or a reduction in the binding affinity of the protein to insecticides) (Narahashi 1988) which, in turn, enhances the insect's resistance to insecticides. Insecticides such as DDT and pyrethroids specifically target the sodium channels in the nervous system (Narahashi 1988; 1996).

Pyrethroids and DDT deliver their toxic, insecticidal effects primarily by binding onto the sodium channel, altering its gating properties and keeping the sodium channel open for unusually longer time, thereby causing a prolonged flow of sodium current. This prolonged sodium current elevates and prolongs the depolarizing phase of the action potential of the neuron membrane, which initiates repetitive discharges and prevents the repolarization phase of action potentials (Narahashi 1988). However, modifications in the sodium channel structure (i.e., point mutation or substitution, resulting from single nucleotide polymorphisms [SNP]) cause insensitivity to DDT and pyrethroids in the sodium channels of the insect's nervous system, via a reduction in or an elimination of the binding affinity of the insecticides to proteins (Narahashi 1988), thus diminishing the toxic effects of the insecticides and resulting in insecticide resistance (Soderlund 2005; Dong 2007). Reduced target-site sensitivity of sodium channels is known to be one of the major mechanisms involved in pyrethroid resistance and is referred to as knockdown resistance (kdr) (Soderlund and Knipple 2003).

As part of our effort to characterize all the mutations in an entire mosquito sodium channel that are involved in pyrethroid resistance, we first cloned and sequenced the full-length of the sodium channel cDNA for $C x$. quinquefasciatus and identified, for the first time, both nonsynonymous and synonymous mutations, including the L-to-F $k d r$ mutation, that are 
co-present in the sodium channel of individual mosquitoes (Xu et al. 2011). With the full length of the sodium channel cDNA clones and a large number of mutations identified, we are now in an excellent position to fully characterize the key mutations contributing to insecticide resistance in an entire mosquito sodium channel, and we plan to investigate the effects of these key mutations and mutation combinations on the sensitivity of the mosquito sodium channel to pyrethroids.

To shed light on the connection between sodium channel mutations and pyrethroid resistance, $\mathrm{Xu}$ et al. (2006a; b) compared genomic DNA and RNA expression levels within the same individuals for mosquitoes $C x$. quinquefasciatus, house flies $M$. domestica, and German cockroaches Blattella germanica bearing different resistant phenotypes ranging from susceptible to highly resistant. In these studies, no correlation for the L-to-F allele at the genomic DNA level with either level of susceptibility or resistance to insecticide was identified in any of the three insect species examined. However, a strong correlation between the L-to-F allele expression and levels of insecticide resistance and susceptibility was observed. These findings offer a completely new approach to exploring how these target site genotypes and their insensitivity-mediated resistance phenotypes are coupled.

Building on the above research, an in-depth investigation of the genomic organization and allelic expression at the L-to-F site of the sodium channel gene in Cx. quinquefasciatus has been conducted (Xu et al. 2011). Multiple copies of the sodium channel gene were identified in the mosquito $C x$. quinquefasciatus by Southern blot analysis and polymerase chain reaction (PCR) analysis (Xu et al. 2011). Two genomic DNA fragments of the mosquito sodium channel gene (509 bp and $181 \mathrm{bp}$ ) were detected by a single PCR primer pair. Sequence analysis indicated the lack of an intron sequence in the $181 \mathrm{bp}$ sodium channel fragment. Single nucleotide polymorphism (SNP) analysis revealed a strong correlation among the frequencies of L-to-F allelic (T) expression at the RNA level, the frequencies and resistance allele (T) at the L-to-F site of the $509 \mathrm{bp}$ genomic DNA fragment (which did include an intron sequence), and the levels of insecticide resistance. This study, for the first time, not only revealed that multiple copies of the sodium channel gene are present in the Culex mosquito genome but also suggested that the copy containing the intron sequence may be a functional copy of the sodium channel gene in Culex mosquitoes. Further investigation of the role of these multiple copies of the sodium channel gene in the genome of mosquitoes will provide a more comprehensive picture of the mechanisms involved in the development of sodium channel mediated pyrethroid resistance in mosquitoes.

\section{Multiple gene interactions in pyrethroid resistance}

Although it is possible that an individual mechanism acting alone could confer resistance, there is a considerable body of evidence indicating that an interaction involving multiple resistance mechanisms or genes is responsible for high levels of insecticide resistance. Results from both our studies and those of many other research groups suggest that the interaction of multiple insecticide resistance mechanisms or genes is likely to be responsible for the development of insecticide resistance. For example, multiple genes have been found to be up-regulated in insecticide resistant HAmCq mosquitoes (Liu et al. 2007). Apart from two of the P450 genes, most of the up-regulated genes had not previously been reported in the literature on insecticide resistance. Two of the overexpressed genes are rhodopsin and arrestin, both of which play a crucial role in signal transduction systems. Rhodopsin is an archetypal class A G-protein-coupled receptor (GPCR) (Filipek et al. 2003). GPCRs act as 
transducers for a range of different sensory, chemotactic, hormonal, and neuronal signals, and are involved in many essential physiological functions of organisms. Arrestins are members of a gene family of regulatory proteins that, along with G-protein-coupled receptor kinases (GRKs) and other co-factors, regulate the signaling and trafficking of Gprotein-coupled receptors by virtue of their preferential binding to the phosphorylated active form of the receptor. Up-regulation of these two genes in resistant mosquitoes may indicate that the neuronal signaling is affected in resistant mosquitoes. The resistancespecific overexpression of arrestins and rhodopsin in resistant mosquitoes (Liu et al. 2007) highlights the functional importance of the signal transduction system in the regulation of insecticide resistance. Similarly, Vontas's group reported multiple genes (including a P450 gene, CYP314A1) to be up-regulated in DDT-resistant Anopheles gambiae mosquitoes (Vontas et al. 2006). While it is unknown whether and how these up-regulated genes are associated with insecticide resistance, the results of these two studies not only indicate a common phenomenon of insecticide resistance conferred by multi-resistance mechanism interactions, but also offer a tantalizing hint of a regulatory relationship among different mechanisms and/or genes in resistance.

\section{The trans and/or cis regulation in pyrethroid resistance}

The regulation of trans and/or cis genes (factors) that account for the same resistance mechanism may provide another explanation for high levels of resistance. Many studies have demonstrated that the up-regulation of P450 and GST genes in resistant insects is regulated by trans and/or cis regulatory genes. The up-regulation of a GST gene (GST-2) in the mosquito Aedes aegypti is controlled by a trans-acting factor (Grant and Hammock 1992), while the up-regulation of 2 P450 genes, CYP6A1 and CYP6D1, in the house fly M. domestica is known to be trans-regulated by one or more factors on autosome 2 (Carino et al. 1994; Liu and Scott 1996). The up-regulation of CYP6A2 and CYP6A8 in the fruit fly Drosophila melanogaster is transcriptionally regulated by trans-regulatory factors (Maitra 2000).

Our group has also recently examined trans and/or cis regulation of insecticide resistance or resistance genes in house flies by characterizing autosomal interactions and contributions, both individually and in combination, and its influence on the development of pyrethroid resistance (Tian et al. 2011). Five $\mathrm{BC}_{1}$ lines and 16 mass-cross homozygous lines were generated from crosses of the pyrethroid resistant ALHF (wild-type) and susceptible aabys (bearing recessive morphological markers on each of five autosomes) strains. The resulting homozygous lines all had combinations of autosomes that differed from those of the resistant ALHF strain. The results indicated that factors on autosome 4 are not involved in the development of resistance in house flies, while factors on autosomes 1, 2, 3 and 5 play important roles in pyrethroid resistance. The sodium channel gene mapped onto autosome 3 and multiple cytochrome P450 genes overexpressed in resistant ALHF house flies were genetically mapped on autosome 5, suggesting that sodium channel-mediated target site insensitivity and P450-mediated detoxification located on autosomes 3 and 5, respectively, are major factors related to the development of resistance in house flies. However, neither of the factors on autosome 3 or 5 alone, nor the factors from both autosomes 3 and 5 combined, could confer the high levels of resistance to pyrethroids actually observed. Strong synergistic effects on resistance are obtained when autosomes 1 and 2 interact with autosome 3 and/or 5 however, suggesting that the trans factors on autosomes 1 and 2 may interact with factors on autosomes 3 and 
5 and thus play a regulatory role in the development of sodium channel insensitivity- and P450 detoxification-mediated resistance.

Taken together, the above findings not only indicate a common phenomenon of insecticide resistance conferred by multi-resistance mechanism interactions, but also offer tantalizing clues suggesting a regulatory relationship among different mechanisms and/or genes in resistance, although none of these regulatory genes has as yet been characterized.

\section{Acknowledgments}

The studies performed by the author's group were supported by Award Numbers R21AI076893 and R21AI090303 to N.L. from the National Institute of Allergy and Infectious Diseases, the U.S. Department of Agriculture National Research Initiative (USDA-NRI) Competitive Grants Program; AAES Hatch/Multistate Grants ALA08-045 and ALA015-110026 to N.L. AAES Foundation Grants; Auburn University and Auburn University Biogrant 2008-Bio-Liu-03-08; the Hatch Project ALA08-029; and the Department of Entomology and Plant Pathology, Auburn University. The author sincerely thanks all the people who have ever worked and are currently working in her laboratory for their hard work and dedication to the field of insecticide resistance research.

\section{References}

Berenbaum, M.R., 1991. Coumarins. In: Rosenthal, G.A., Berenbaum, M.R. (Eds.), Herbivores: Their Interaction with Secondary Plant Metabolites, Academic Press, New York, pp. 221-249.

Butler, D., 2011. Mosquitoes score in chemical war. Nature 475, 19-20.

Carino, F.A., Koener, J.F., Plapp, F.W., Jr., Feyereisen, R., 1994. Constitutive overexpression of the cytochrome P450 gene CYP6A1 in a house fly strain with metabolic resistance t0 insecticides. Insect Biochem. Mol. Biol. 24, 411-418.

Dong, K., 2007. Insect sodium channels and insecticide resistance. Invert. Neurosc. 7, 17-30. Feyereisen, R. 1995, Toxicol. Lett., 82/83, 83-90.

Feyereisen, R., 2005. Insect cytochrome P450. In: Gilbert, L.I., Iatrou, K., Gill, S. (Eds.), Comprehensive Molecular Insect Science, vol. 4. Elsevier, Oxford, pp. 1-77.

Filipek, S., Stenkamp, R.E., Teller, D.C., Palczewski, K., 2003. G protein-coupled receptor rhodopsin: a prospectus. Annu. Rev. Physiol. 65, 851-879.

Grant, D.F., Hammock, B.D., 1992. Genetic and molecular evidence for a trans-acting regulatory locus controlling glutathione S-tranferase-2 expression in Aedes aegypti. Mol. Gen. Genet. 234, 169-176.

Gonzalez, F.J., Nebert, D.W., 1990. Evolution of the P450 gene superfamily: animal-plant "warfare", molecular drive, and human genetic differences in drug oxidation. Trends Genet. 6, 182-186.

Hemingway, J., Field, L., Vontas, J., 2002. An overview of insecticide resistance. Science 298, 96-97.

Hemingway, J., Hawkes, N.J., McCarroll, L., Ranson, H., 2004. The molecular basis of insecticide resistance in mosquitoes. Insect Biochem. Mol. Biol. 34, 653-665.

Hemingway, J., Karunaratne, S.H., 1998. Mosquito carboxylesterases: a review of the molecular biology and biochemistry of a major insecticide resistance mechanism. Med. Vet. Entomol. 12, 1-12. 
Hodgson, E., 1985. Mocrosomal mono-oxygenases. In: Kerkut, G.A., Gilbert, L.I. (Eds.), Comprehensive Insect Physiology, Biochemistry and Pharmacology, vol. 11. Pergamon, Oxford, pp. 225-321.

Komagata, O., Kasai, S., Tomita, T., 2010. Overexpression of cytochrome P450 genes in pyrethroid-resistant Culex quinquefasciatus. Insect Biochem. Mol. Biol. 40, 146-152.

Li, T., Liu, N., 2010. Genetics and Inheritance of Permethrin Resistance in the Mosquito Culex quinquefasciatus. J. Med. Entomol. 47, 1127-1134.

Li, W., Petersen, R.A., Schuler, M.A., Berenbaum, M. R., 2002. CYP6B cytochrome P450 monooxygenases from Papilio canadensis and Papilio glaucus: potential contributions of sequence divergence to host plant associations. Insect Mol. Biol. 11, 543-551.

Liu, H., Cupp, E.W., Micher, K.M., Guo, A., Liu, N., 2004a. Insecticide resistance and crossresistance in Alabama and Florida strains of Culex quinquefasciatus. J Med. Entom. 41, 408-413.

Liu, H., Cupp, E.W., Guo, A., Liu, N., 2004b. Insecticide resistance in Alabama and Florida mosquito strains of Aedes albopictus. J. Med. Entom. 41, 946-952.

Liu, N., Li, T., Reid, W.R., Yang, T., Zhang, L., 2011. Multiple cytochrome P450 genes: their constitutive overexpression and permethrin induction in insecticide resistant mosquitoes, Culex quinquefasciatus. PlosOne.

Nelson, D.R., 2009. The Cytochrome P450 Homepage. Human Genom. 4, 59-65.

Liu, N., Liu, H., Zhu, F., Zhang, L., 2007. Differential expression of genes in pyrethroid resistant and susceptible mosquitoes, Culex quinquefasciatus. Gene. 394, 61-68.

Liu, N., Scott, J.G., 1995. Genetics of resistance to pyrethroid insecticides in the house fly, Musca domestica. Pestic. Biochem. Physiol. 52, 116-124.

Liu, N., Scott, J.G., 1996. Genetic analysis of factors controlling elevated cytochrome P450, CYP6D1, cytochrome b5, P450 reductase and monooxyenase activities in LPR house flies, Musca domestica. Biochem. Genet. 34, 133-148.

Liu, N., Scott, J.G., 1997. Inheritance of CYP6D1-mediated pyrethroid resistance in house fly (Diptera: Muscidae). J. Econ. Entomol. 90, 1478-1481.

Liu, N., Scott, J.G., 1998. Increased transcription of CYP6D1 causes cytochrome P450mediated insecticide resistance in house fly. Insect Biochem. Mol. Biol. 28, 531-535.

Liu, H., Xu, Q., Zhang, L., Liu, N., 2005. Chlorpyrifos resistance in the mosquito Culex quinquefasciatus. J. Med. Entomol. 42, 815-820.

Liu, N., Yue, X., 2000. Insecticide resistance and cross-resistance in the house fly (Diptera: Muscidae). J. Econ. Entomol. 93, 1269-1275.

Liu, N., Yue, X., 2001. Genetics of pyrethroid resistance in a strain (ALHF) of house flies (Diptera: Muscidae). Pestic. Biochem. Physiol. 70, 151-158.

Maitra, S., Dombrowski, S.M., Basu, M., et al., 2000. Factors on the third chromosome affect the level of Cyp6a2 and Cyp6a8 expression in Drosophila melanogaster. Gene. 248,147-156.

Maitra, S., Dombrowski, S.M., Waters, L.C., Ganguly, R., 1996. Three second chromosomelinked clustered Cyp6 genes show differential constitutive and barbital-induced expression in DDT-resistant and susceptible strains of Drosophila melanogaster. Gene 180, 165-171.

Narahashi, T., 1988. Molecular and cellular approaches to neurotoxicology: past, present and future. In: Lunt, G.G. (Ed.), Neurotox '88: Molecular Basis of Drug and Pesticide Action, Elsevier, New York, pp. 563-582. 
Narahashi, T., 1996. Neuronal ion channels as the target sites of insecticides. Pharmacol. Toxicol. 78, 1-14.

Oakeshott, J.G., Claudianos, C., Campbell, P.M., Newcomb, R.D., Russell, R.J., 2005. In: Gilbert, L.I., Iatrou, K., Gill, S. (Eds.), Biochemical genetics and genomics of insect esterases. Comprehensive Molecular Insect Science, vol. 5. Elsevier, Oxford, pp. 309-381.

Okey, A.B., 1990. Enzyme induction in the cytochrome P-450 system. Pharmacol. Ther. 45, 241-298.

Ortelli, F., Rossiter, L.C., Vontas, J., Ranson, H., Hemingway, J., 2003. Heterologous expression of four glutathione transferase genes genetically linked to a major insecticide-resistance locus from the malaria vector Anopheles gambiae. Biochem. J. 373, 957-963.

Pasteur, N., Raymond, M., 1996. Insecticide resistance genes in mosquitoes: their mutations, migration, and selection in field populations. J. Hered. 87, 444-449.

Pavek, P., Dvorak, Z., 2008. Xenobiotic-induced transcriptional regulation of xenobiotic metabolizing enzymes of the cytochrome P450 superfamily in human extrahepatic tissues. Curr. Drug Metab. 9, 129-143.

Ranson, H., Claudianos, C., Ortelli, F., Abgrall, C., Hemingway, J., Sharakhova, M.V., Unger, M.F., Collins, F.H., Feyereisen, R., 2002. Evolution of supergene families associated with insecticide resistance. Science 298, 179-181.

Ranson, H., Hemingway, J., 2005. Glutathione transferases. In: Gilbert, L.I., Iatrou, K., Gill, S. (Eds.), Comprehensive Molecular Insect Science, vol. 5. Elsevier, Oxford, pp. 383402.

Raymond, M., Arnaouty, V.B., Sivasubramanian, N., Mouches, C., Georghiou, G.P., Pasteur, N., 1989. Amplification of various esterase B's responsible for organophosphate resistance in Culex mosquitoes. Biochem. Genet. 27, 417-423.

Schuler, M.A., 1996. The role of cytochrome P450 monooxygenases in plant-insect interactions. Plant Physiol. 112, 1411-1419.

Scott, J.G., 1990 Investigating mechanisms of insecticide resistance: methods, strategies and pitfalls. In: Roush R.T., Tabashnik B.E. (eds.), Pesticide Resistance in Arthropods, Chapman and Hall, New York, pp. 39-57.

Scott, J.G., 1999. Cytochromes P450 and insecticide resistance. Insect Biochem. Mol. Biol. 29, 757-777.

Scott, J.G., Liu N., Wen, Z., 1998. Insect cytochrome P450: diversity, insecticide resistance and tolerance to plant toxins. Comp. Biochem. Physiol. 121C, 147-155.

Small, G.J., Hemingway, J., 2000. Molecular characterization of the amplified carboxylesterase gene associated with organophosphorus insecticide resistance in the brown planthopper, Nilaparvata lugens. Insect Mol. Biol. 9, 647-653.

Soderlund, D.M., 2005. Sodium channels. In: Gilbert, L.I., Latrou K. and Gill, S.S. (Eds.), Comprehensive Molecular Insect Science, vol. 5. Elsevier BV, Oxford, pp. 1-24.

Soderlund, D.M., Knipple, D.C., 2003. The molecular biology of knockdown resistance to pyrethroid insecticides. Insect Biochem. Mol. Biol. 33, 563-577.

Terriere, L.C., 1983. Enzyme induction, gene amplification, and insect resistance to insecticides. In: Georghiou, G.P., Saito, T. (Eds.), Pest Resistance to Pesticides. Plenum Press, New York, pp. 265-297. 
Terriere, L.C., 1984. Induction of detoxication enzymes in insects. Ann. Rev. Entomol. 29, 7188.

Vontas, J., Blass, C., Koutsos, J.A.C., David, P., Kafatos, F.C., Louis, C., Hemingway, J., Christophides, G.K., Ranson, H., 2005. Gene expression in insecticide resistant and susceptible Anopheles gambiae strains constitutively or after insecticide exposure. Insect Mol. Biol. 14, 509-521.

Wen, Z., Pan, L., Berenbaum, M.B., Schuler, M.A., 2003. Metabolism of linear and angular furanocoumarins by Papilio polyxenes CYP6B1 co-expressed with NADPH cytochrome P450 reductase. Insect Biochem. Mol. Biol. 33, 937-947.

Whyard, S., Downe, A.E.R., Walker V. K., 1995. Characterization of a novel esterase conferring insecticide resistance in the mosquito Culex tarsalis. Arch. Insect Biochem. Physiol. 29, 329-342.

World Health Organization. 1957. Expert committee on insecticides. W.H.O. Tech. Rpt. Ser. 7th Rpt.

Xu, Q., Liu, H., Zhang, L., Liu, N., 2005. Resistance in the mosquito, Culex quinquefasciatus (S.), and possible mechanisms for resistance. Pest Manag. Sci 61, 1096-1102.

$\mathrm{Xu}$, Q., Wang, H., Zhang, L., Liu, N., 2006a. Kdr allelic variation in pyrethroid resistant mosquitoes, Culex quinquefasciatus (S.). Biochem. Biophys. Res. Commun. 345, 774780 .

Xu, Q., Wang, H., Zhang, L., Liu, N., 2006b. Sodium channel gene expression associated with pyrethroid resistant house f lies and German cockroaches. Gene 379, 62-67.

Xu, Q., Tain, L., Zhang, L., Liu, N., 2011. Sodium channel genes and their differential genotypes at the L-to-F $k d r$ locus in the mosquito Culex quinquefasciatus. Biochem. Biophys. Res. Com. 407, 645-649.

Zaim, M., Guillet, P., 2002. Alternative insecticides: an urgent need. Trends Parasitol. 18, 161-163.

Zhu, F., Feng, J., Zhang, L., Liu, N., 2008a. Characterization of two novel cytochrome P450 genes in insecticide-resistant house-flies. Insect Mol. Biology 17, 27-37.

Zhu, F., Li, T., Zhang, L., Liu, N., 2008b. Co-up-regulation of three P450 genes in response to permethrin exposure in permethrin resistant house flies, Musca domestica. BMC Physiol. 8: 18.

Zhu, F., Liu, N., 2008. Differential expression of CYP6A5 and CYP6A5v2 in pyrethroidresistant house flies, Musca domestica. Arch. Insect Biochem. Physiol. 67, 107-119. 


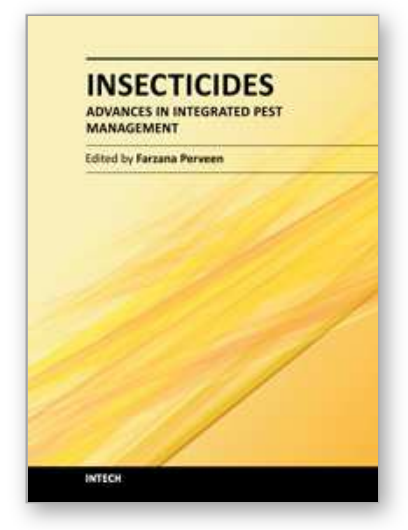

\author{
Insecticides - Advances in Integrated Pest Management \\ Edited by Dr. Farzana Perveen
}

ISBN 978-953-307-780-2

Hard cover, 708 pages

Publisher InTech

Published online 05, January, 2012

Published in print edition January, 2012

This book contains 30 Chapters divided into 5 Sections. Section A covers integrated pest management, alternative insect control strategies, ecological impact of insecticides as well as pesticides and drugs of forensic interest. Section B is dedicated to chemical control and health risks, applications for insecticides, metabolism of pesticides by human cytochrome $\mathrm{p} 450$, etc. Section $\mathrm{C}$ provides biochemical analyses of action of chlorfluazuron, pest control effects on seed yield, chemical ecology, quality control, development of ideal insecticide, insecticide resistance, etc. Section $D$ reviews current analytical methods, electroanalysis of insecticides, insecticide activity and secondary metabolites. Section E provides data contributing to better understanding of biological control through Bacillus sphaericus and B. thuringiensis, entomopathogenic nematodes insecticides, vector-borne disease, etc. The subject matter in this book should attract the reader's concern to support rational decisions regarding the use of pesticides.

\title{
How to reference
}

In order to correctly reference this scholarly work, feel free to copy and paste the following:

Nannan Liu (2012). Pyrethroid Resistance in Insects: Genes, Mechanisms, and Regulation, Insecticides Advances in Integrated Pest Management, Dr. Farzana Perveen (Ed.), ISBN: 978-953-307-780-2, InTech, Available from: http://www.intechopen.com/books/insecticides-advances-in-integrated-pestmanagement/pyrethroid-resistance-in-insects-genes-mechanisms-and-regulation

\section{INTECH}

open science | open minds

\author{
InTech Europe \\ University Campus STeP Ri \\ Slavka Krautzeka 83/A \\ 51000 Rijeka, Croatia \\ Phone: +385 (51) 770447 \\ Fax: +385 (51) 686166 \\ www.intechopen.com
}

\author{
InTech China \\ Unit 405, Office Block, Hotel Equatorial Shanghai \\ No.65, Yan An Road (West), Shanghai, 200040, China \\ 中国上海市延安西路65号上海国际贵都大饭店办公楼 405 单元 \\ Phone: +86-21-62489820 \\ Fax: $+86-21-62489821$
}


(C) 2012 The Author(s). Licensee IntechOpen. This is an open access article distributed under the terms of the Creative Commons Attribution 3.0 License, which permits unrestricted use, distribution, and reproduction in any medium, provided the original work is properly cited. 